\title{
Approach to native medical renal biopsy interpretation of glomerular disease
}

\section{Gopi Aryal ${ }^{1}$, Sameer Chhetri Aryal ${ }^{2}$}

${ }^{I}$ Department of Laboratory Medicine \& pathology, Mediciti Hospital, Lalitpur, Nepal

${ }^{2}$ Henry Ford Health System, Michigan, USA

\section{Keywords:}

Electron microscopy; Glomerulonephritis; Immunohistology;

Renal biopsy;

\begin{abstract}
Diverse pathogenetic mechanisms and clinical manifestation of renal diseases may produce the same renal morphologic pattern or variety of renal morphologic pattern can lead to the same clinical syndrome. The primary role of the renal biopsy is to provide a diagnosis and information about disease activity and chronicity.

The systematic approach to native medical renal biopsy includes evaluation of the four compartments of the kidney sequentially (glomeruli, tubules, interstitium, and blood vessels). The diagnosis in renal pathology is an integrated process in which we must analyze all clinical data, light microscopy, immunohistology, and electron microscopy studies for diagnosis.

The aim of the article is to describe the handling of the renal tissue in the anatomical pathology laboratory. It also provides the guideline to renal biopsy evaluation and approach to arrive at the diagnosis of glomerular diseases with similar clinical presentations.
\end{abstract}

\section{Correspondence:}

Dr. Gopi Aryal, MD, PhD

Chair, Department of Laboratory Medicine \& Pathology

Nepal Mediciti Hospital, Pvt. Ltd., Nepal

ORCID ID: 0000-0002-8566-2593

Email: gopiaryal1@gmail.com

Received : August $15^{\text {th }} 2018$; Accepted : September $16^{\text {th }} 2019$

Citation: Aryal G, Aryal SC. Approach to native medical renal biopsy interpretation of glomerular disease. J Pathol Nep 2019;9:1571-80 DOI 10.3126/jpn.v9i2.25664

Copyright: This is an open-access article distributed under the terms of the Creative Commons Attribution 4.0 International License, which permits unrestricted use, distribution, and reproduction in any medium, provided the original author and source are credited.

\section{INTRODUCTION}

The renal biopsy is essential to distinguish among glomerular diseases with similar clinical presentations. Diverse pathogenetic mechanisms may produce a similar morphologic response. The primary role of the renal biopsy is to provide a diagnosis and information about disease activity and chronicity. Most of the glomerular injuries are thought to be immune-mediated. ${ }^{1,2}$

In the majority of cases, the inciting etiologic agents remain unknown with the exception of glomerulonephritis (GN) that are clearly induced by infections, such as post streptococcal 
and hepatitis $\mathrm{C}$ virus-induced cryoglobulinemic $\mathrm{GN}$, and a few examples of drug-induced and cancer-associated GNs. ${ }^{3,4}$ Glomerular injury is usually mediated by the actions of multiple elements of both the innate and the adaptive immune systems, resulting in diverse clinical and pathologic manifestations. ${ }^{5}$ The type of injury and the renal host response are important in defining the morphologic manifestations and in determining the clinical course of the patient.

\section{ULTRASTRUCTURE OF GLOMERULUS}

The layers (inner to outer) of glomeruli are a) Fenestrated endothelium; b) Glomerular Basement membrane; c) Podocytes (visceral epithelium with foot processes). These foot processes are embedded in lamina rara externa of the glomerular basement membrane and have filtration slits of 70-100nm dimension between the foot processes. The fourth component is parietal epithelium which lines Bowman's space and the last component is mesangial cells.

\section{SITE OF GLOMERULAR INJURY}

The major determinant of whether the patient presents with nephritic or with nephrotic syndrome is the site of glomerular injury. There are three major types of resident glomerular cells: epithelial cells, endothelial cells, and mesangial cells. Glomerular endothelial and mesangial cells come into direct contact with circulating factors like complement and inflammatory cells including neutrophils, macrophages, natural killer cells, and $\mathrm{T}$ cells. Immune complexes that form or deposit in the mesangial matrix or subendothelial space often result in glomerular inflammation and GN. Glomerular visceral epithelial cells or podocytes are the major barrier that restricts the filtration of negatively charged or large $(>60 \mathrm{kDa})$ plasma proteins. Injury to podocytes leads to massive proteinuria and is often associated with the nephrotic syndrome. As podocytes are separated from the circulation by the glomerular basement membrane (GBM), subepithelial immune deposits that target podocytes seldom produce glomerular inflammation, and therefore there is little or no hematuria and no red blood cell casts.

In response to glomerular injury, there is an increase in glomerular permeability to macromolecules such as transforming growth factor-beta, fatty acids, proinflammatory markers of oxidant stress, and protein may result in toxicity to the mesangial matrix, causing mesangial cell expansion, inflammation, fibrosis, and glomerular scarring. Both the structural alterations and accompanying biochemical, cellular, and molecular changes seem to account for progressive renal scarring and loss of kidney function.

\section{CLINICAL MANIFESTATION OF RENAL DISEASE}

Following are the clinical manifestations of renal diseases:

- Selective proteinuria

- Non-selective proteinuria

- Nephrotic-range proteinuria

- Nephrotic syndrome

- Nephritic syndrome

- Isolated glomerular hematuria

- Rapidly progressive glomerulonephritis (RPGN)

- Hypertension

- Thrombotic microangiopathy

- Acute kidney injury

- Chronic Kidney disease

- Urinary tract infection

\section{EVALUATION OF PROTEINURIA}

Average daily urinary protein excretion in adults is $80 \mathrm{mg} /$ day, with normal excretion considered to be $<150 \mathrm{mg} /$ day. Albumin represents approximately $15 \%$ of the daily urinary protein excretion in healthy people, with other plasma proteins (e.g., immunoglobulins, beta-2-microglobulin) and Tamm-Horsfall protein constituting the remaining $85 \%$. Proteinuria varies in amount and may be transient or persistent. ${ }^{6,7}$

It is important to distinguish persistent and transient proteinuria. The common causes for transient proteinuria are fever, heavy physical exertion, urinary tract infection, orthostatic proteinuria and pregnancy. The presence of dipstick-positive proteinuria on 2 urine samples separated by 1 to 2 weeks indicates persistent proteinuria and warrants quantification and further evaluation. ${ }^{8}$ If orthostatic proteinuria is suspected, the protein-to-creatinine ratio in a first morning voided urine specimen should be compared with the ratio from a random sample later in the day. The absence of proteinuria in the morning sample and presence in the daytime sample confirms orthostatic proteinuria. The presence of persistent proteinuria should prompt evaluation of kidney function by estimation of GFR from serum creatinine and/or collection of 24-hour urine for creatinine clearance. Urgent consideration is required in proteinuria 
Table 1: Indications for renal Biopsy

\begin{tabular}{ll}
\hline Indications & Comments \\
\hline Hematuria & Presence of acanthocytes or red blood cell casts with an elevated Scr level or proteinuria \\
Proteinuria & $\begin{array}{l}\text { Proteinuria }>1 \mathrm{~g} / \mathrm{d} \text { as measured on multiple visits with no clear comorbidity; proteinuria }>3 \mathrm{~g} / \mathrm{d} \text { in the absence of } \\
\text { diabetes or a rapid increase in proteinuria even with diabetes; proteinuria }<3 \mathrm{~g} / \mathrm{d} \text { with an elevated Scr level with no clear } \\
\text { comorbid conditions such as diabetes or hypertension }\end{array}$ \\
\hline AKI & $\begin{array}{l}\text { In the setting of ATI, persistent injury despite reversal of cause or if Scr did not return to baseline with 7-14 d of injury } \\
\text { onset; in the setting of presumptive AIN, if there has been no resolution of injury despite removal of culprit medication }\end{array}$ \\
\hline CKD & Rapid elevation in Scr level or new-onset hematuria or proteinuria \\
\hline
\end{tabular}

Abbreviations: AIN, acute interstitial nephritis; AKI, acute kidney injury; ATI, acute tubular injury; CKD, chronic kidney disease; Scr, serum creatinine.

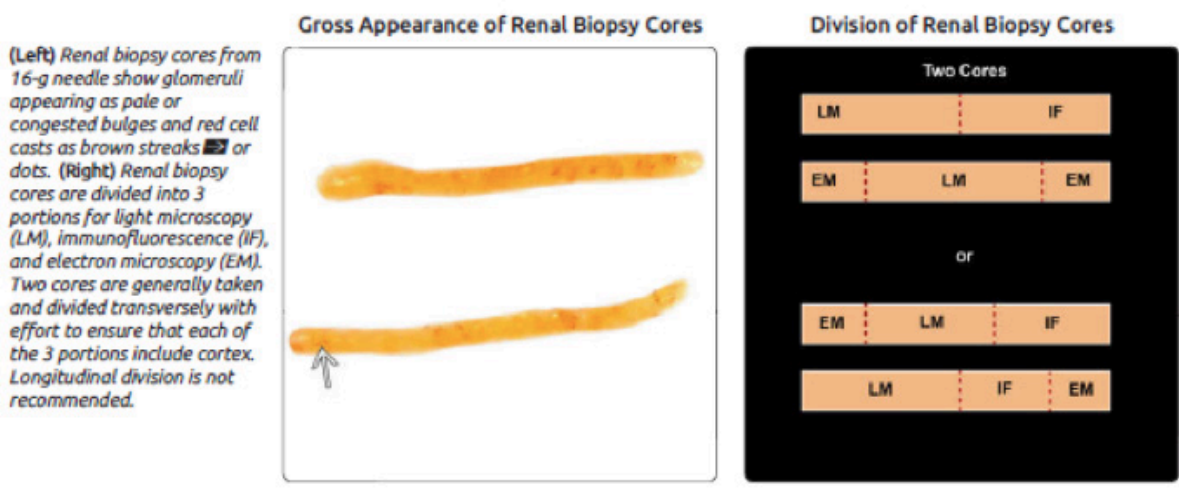

Figure 1: Renal biopsy cores

due to renal vein thrombosis or features with RPGN.

\section{INDICATION FOR RENAL BIOPSY}

A kidney biopsy is required to make a definitive diagnosis that might affect treatment or provide information about disease progression or prognosis. A biopsy should be avoided when the potential risk to the patient exceeds any likely benefit from procuring kidney tissue. Indication for a renal biopsy is summarized in Table $1 .{ }^{9}$

Percutaneous renal biopsy for the detection of primary renal disease is generally not pursued in the following settings:

- Small hyperechoic kidneys $(<9 \mathrm{~cm})$, which are generally indicative of chronic irreversible disease

- Solitary native kidney

- Multiple, bilateral cysts or a renal tumor

- Uncorrectable bleeding diathesis

- Severe hypertension that cannot be controlled with antihypertensive medications

- Hydronephrosis
- Active renal or perirenal infection

- Anatomic abnormalities of the kidney that may increase risk (see above)

- Skin infection over the biopsy site

- An uncooperative patient

Tissue sampling and preparation

Percutaneous ultrasound-guided kidney biopsy is usually performed on the inferior pole of the left or right kidney. The left kidney is visible without other organs impeding the acquisition of the tissue. An adequate assessment of native renal biopsies includes light microscopy (LM), immunofluorescence microscopy (IF), and electron microscopy (EM).

\section{The Biopsy Core}

The specimen should be handled very carefully to avoid squeezing and compression, preferably by wooden sticks instead of forceps. A needle biopsy specimen should be divided under a dissecting microscope. This microscope allows for visualization and differentiation of the renal cortex and medulla. The medulla shows parallel structures 
Table 2: Terms used to describe histologic lesions in glomeruli. ${ }^{12}$

\begin{tabular}{|c|c|}
\hline \multicolumn{2}{|l|}{ Distributional descriptors } \\
\hline Focal & Involving $<50 \%$ of glomeruli \\
\hline Diffuse & Involving $50 \%$ or more of glomeruli \\
\hline Segmental & Involving part of a glomerular tuft \\
\hline Global & Involving all of a glomerular tuft \\
\hline Lobular & Appearance due to endocapillary hypercellularity \\
\hline Nodular & Relatively acellular, round areas of mesangial matrix expansion \\
\hline Membranoproliferative & Combined capillary wall thickening with double contours and mesangial or endocapillary hypercellularity \\
\hline Mesangium & Stalk region of capillary loop with mesangial cells surrounded by matrix \\
\hline \multicolumn{2}{|l|}{ Lesional descriptors } \\
\hline Membranous thickening & Global thickening of peripheral capillary walls with spikes \\
\hline Wire loop & Thick, rigid appearance of capillary loop due to massive subendothelial deposits \\
\hline Tram-track & Double contour of glomerular basement due to deposits and/or circumferential interposition \\
\hline Mesangial hypercellularity & Four or more nuclei in a peripheral mesangial segment \\
\hline $\begin{array}{l}\text { Endocapillary hypercellu- } \\
\text { larity }\end{array}$ & Increased cellularity internal to the GBM composed of leukocytes, endothelial cells, and/or mesangial cells \\
\hline $\begin{array}{l}\text { Extracapillary hypercellu- } \\
\text { larity (crescents) }\end{array}$ & Increased cellularity in Bowman's space \\
\hline $\begin{array}{l}\text { Sclerosis (focal and/or } \\
\text { segmental) }\end{array}$ & Increased extracellular matrix expanding the mesangium and obliterating capillary lumen \\
\hline Necrosis & Destruction of cells and matrix with deposition of fibrin \\
\hline Mesangiolysis & Loss of mesangial architecture with lysis of mesangial matrix and loss of mesangial cells \\
\hline Hyaline & Glassy eosinophilic extracellular material \\
\hline
\end{tabular}

(vasa recta and collecting ducts); on the other hand, the cortex appears irregular, and its glomeruli usually are visible as small red capillary conglomerates. In standard preparation, 2 biopsy cores are taken and divided for LM, IF and EM into the appropriate fixative. It needs two small ( $2 \mathrm{~mm}$ ) pieces with glomeruli for EM, a small (3-5mm) piece with glomeruli for IF, and the remaining tissue for paraffin sections for LM. If no dissecting microscope is available, the best method is to cut $1 \mathrm{~mm}$ pieces from both ends of the biopsy specimen for EM and two $2 \mathrm{~mm}$ pieces for IF and submit the rest for routine paraffin processing for LM. Lacking a dissecting microscope, the operator may elect to section each biopsy sample into halves for IF and LM after removing small sections of each for EM from ends. Biopsy cores should contain cortical tissue. Figure 1 demonstrates a method for dividing 2 cores for LM, IF and EM. ${ }^{10}$

\section{RENAL BIOPSY EVALUATION}

Usually, one renal pathologist is involved in providing renal pathology services when the renal biopsy is $<200 /$ year.

Tissue for light microscopy (LM) is processed, dehydrated, and placed in a paraffin block, and multiple serial sections $(2-3 \mu \mathrm{m})$ are obtained and stained. Minimum stains for native renal biopsies are $\mathrm{H} \& \mathrm{E}$ stain with at least two levels, Periodic acid -Schiff (PAS), Silver Methenamine, Masson trichome, and Congo red. Additional unstained slides are produced to allow additional special studies as needed. Five hours of processing, sectioning, and staining time are typically needed to produce LM slides.

\section{Immunofluorescence Microscopy}

Tissue for immunofluorescence (IF) is surrounded with OCT compound and frozen, and sections $(3-4 \mu \mathrm{m})$ are produced and stained with fluorescein-tagged antibodies against $\mathrm{IgG}$, IgA, IgM, C3, C1q, kappa, and lambda light chains. The dilution of antibodies is 1:50. Special studies, including evaluation of serum amyloid A deposits, IgG subclasses (IgG1-4), phospholipase A2 receptor (PLA2R), THSD7A, DNAJB9 and collagen chains (alpha 3.4 and 5), may be helpful in some cases where available. Complement product C4d may also be stained on frozen tissue in allograft biopsy. One to 2 hours of processing, sectioning, and staining time are needed for the production of IF slides.

Reagents required for IF are:

- $\quad$ Michel Transport Media

- $\quad$ Phosphate buffer saline (PBS) pH 7.4

- $\quad$ Buffered Glycol

- $\quad$ PBS washing buffer

\section{Methods}

- Cut $4 \mu \mathrm{m}$ thick section of snap frozen biopsies in a Cryostat set at -25.C (five slides are needed for each 


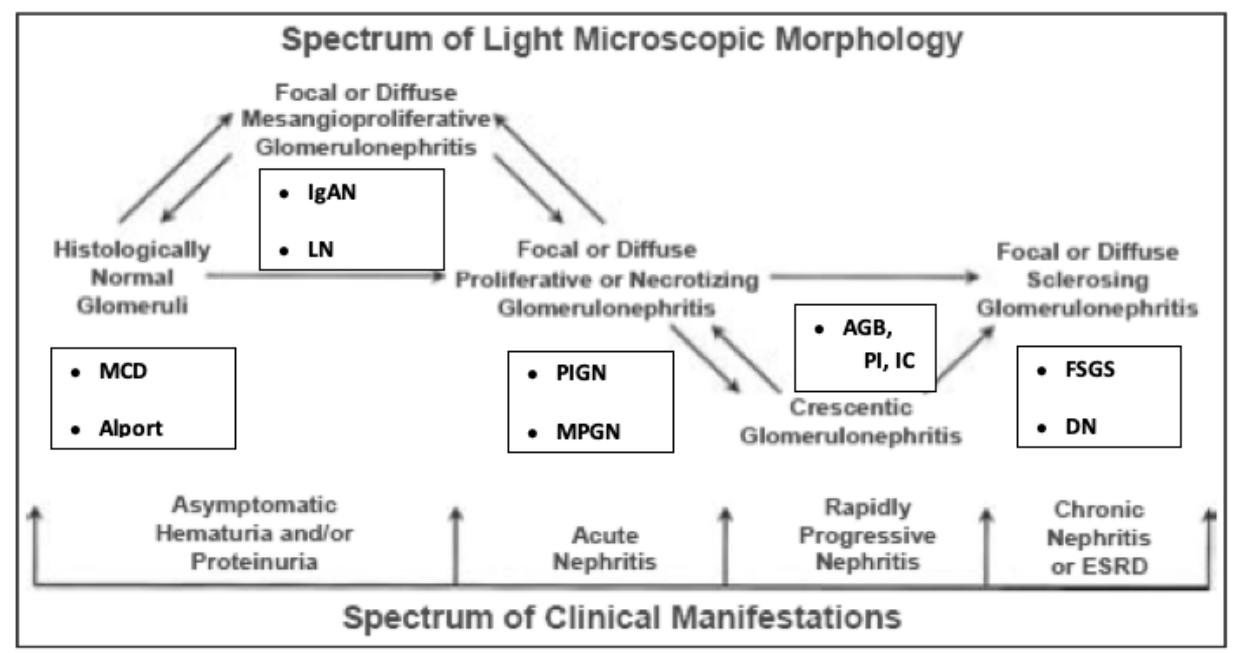

Figure 2: Correlation of various histopathologic patterns of glomerular response to injury. MCD: Minimal Change Disease; IgAN: IgA nephropathy; PIGN: post infectious GN; MPGN: membranoproliferative GN; DN: diabetic nephropathy; FSGS: Focal segmental glomerulosclerosis; AGBM: Anti GBM GN; PI/IC: Pauci immune/immune complex GN; LN: lupus nephritis; ESRD: End stage renal disease

\section{biopsy)}

- Dry section with an electric fan for 10 minutes

- Wash sections in PBS solution for 10 minutes

- $\quad$ Fan dry sections for 10 minutes

- Covers sections of each slide with one of the FITClabeled antisera (1:50 dilution)

- Incubate in a moist chamber at 37.C for $1 \mathrm{hr}$

- Wash section in PBS solution for 10 minutes

- Fan dry sections, mount in buffered glycerol and examine under a fluorescence microscope.

\section{IF on Paraffin sections}

- Cut 3-4 um sections on Poly-L-Lysin coated slides and do the heat fixing as per lab protocols.

- Keep the slides at $60^{\circ} \mathrm{C}$ for $15 \mathrm{~m}$

- Deparaffinize the sections

- Xylene I-10 m

- Xylene II-10 min

- Xylene III-5min

- Ethanol (100\%)-5 min

- Ethanol (70\%)-5 min
- $\quad$ Ethanol (50\%)-5min

- Rinse the sections in distilled water (20 dips) and keep the slides in PBS (pH7.2-7.4)

- Incubate the slides in Tris-EDTA buffer (pH9.0-9.2) at $37^{\circ} \mathrm{C}$ for $30 \mathrm{~min}$.

- Take out the slides from jars and add proteinase $\mathrm{K}$ solution onto the section followed by incubation at 37 ${ }^{0} \mathrm{C}$ for $5 \mathrm{~min}$.

- Stop the digestion by putting above slides in jar containing Tris-EDTA buffer (pH 9.0-9.2) at $4^{\circ} \mathrm{C}$ for 45 $\min$

- Keep the slides in PBS solution for 10 min to start IF staining

- Add FITC conjugated polyclonal antibodies directed against IgG, IgA, IgM, C3, Kappa, Lambda, and C1q as per the dilution recommended by manufacturer. Slight higher dilution as compared to routine IF is recommended for paraffin on IF. Always use freshly prepared antibodies.

- Incubate the slides in humidified chamber at room temperature for 1-2 $\mathrm{hrs}$.

- $\quad$ Rinse the slides with PBS (pH7.2-7.4) for $5 \mathrm{~min}$.

- Mount in aqueous mounting media (PBG, phosphate buffer glycerin)

- Examine the slide under a dark field ultraviolet microscope 


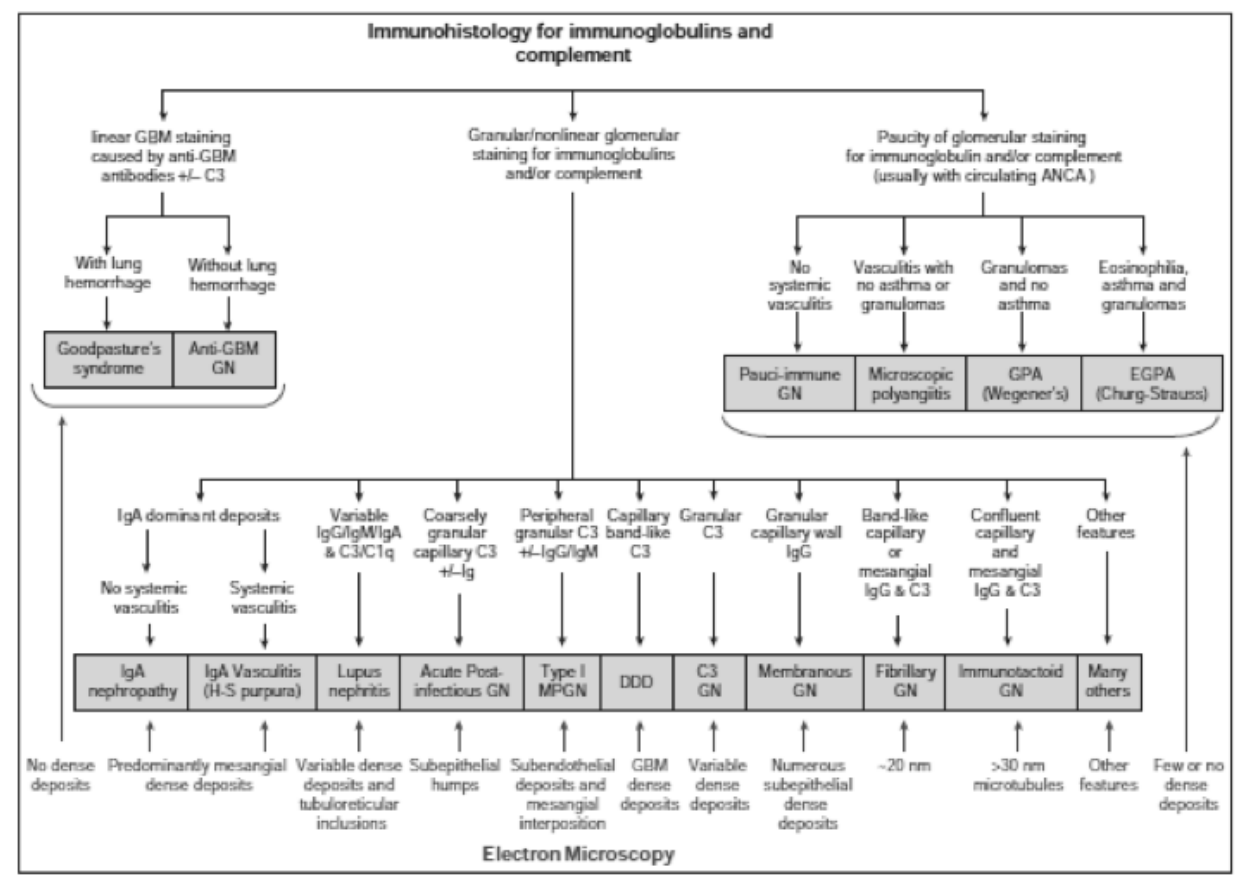

Figure 3: Algorithm for diagnosing immune-mediated glomerular diseases based on the integration of IFM, EM, serology, and other laboratory and clinical data

\section{Electron Microscopsy}

EM tissue is processed and embedded in a plastic, hard media, and thick sections are stained with Toluidine blue to identify the specific area to be cut for thin sections to be placed on a grid for EM examination. Typically 2 working days are needed to process and produce EM sections for ultrastructural examination.

The need for EM should be assessed on the basis of LM findings. If EM technical services are being provided remotely by a specialized diagnostic center, then the semi thin sections from the EM block and the digital EM images should be provided to the pathologist responsible for reporting the renal biopsy.

\section{THE RENAL BIOPSY INTERPRETATION}

The LM, IF and EM findings of renal biopsy should ideally be reported by the same pathologist. Reporting each in isolation may result in serious misdiagnosis. The biopsy report for non-neoplastic kidney diseases represents a complex integration of clinical data with LM, IF and EM findings.

\section{Light Microscopy}

Small samples are adequate for diffuse diseases. Large samples are needed for focal diseases. The minimum sample size for diagnosis varies greatly with the specific diagnosis; for instance, membranous glomerulonephritis can be diagnosed from a single glomerulus. The number of glomeruli required for native renal biopsy for adequacy is 15 to 20 glomeruli. For focal lesions involving a small number of glomeruli, 25 glomeruli may be needed. For renal allograft biopsies, a minimum of 8 glomeruli and two arteries are needed.

Four renal components (glomeruli, tubules, interstitium, and blood vessels) are evaluated sequentially. It should be decided which compartment of the kidney is the most severely involved (primary involvement) along with less prominent changes in other compartments (secondary involvement).

The authors recommend evaluating HE and PAS stained slides under low magnification moving slides back and forth at least seven times even if it appears unremarkable. The first component in the interpretation of glomerular disease is to access glomerular cellularity. Normocellularity is defined as no more than three cells in an individual glomerular mesangial region, away from the vascular pole. If the glomerular hypercellularity is severe and leads to closure of the glomerular capillaries, one has to decide whether the hypercellularity is within the glomerular tuft (intracapillary proliferative GN) or outside the glomerular tuft in Bowman space (extracapillary proliferative GN/crescentic GN). There is evidence that the hypercellularity can result not only from the proliferation of native glomerular cells (i.e., mesangial, endothelial, and epithelial cells) but also from infiltration of mononuclear and/or polymorphonuclear leukocytes.

Glomerular, tubular, and vascular relationships are important. The afferent arterioles perfuse the glomerulus directly, and the cortical tubules are supplied by post 


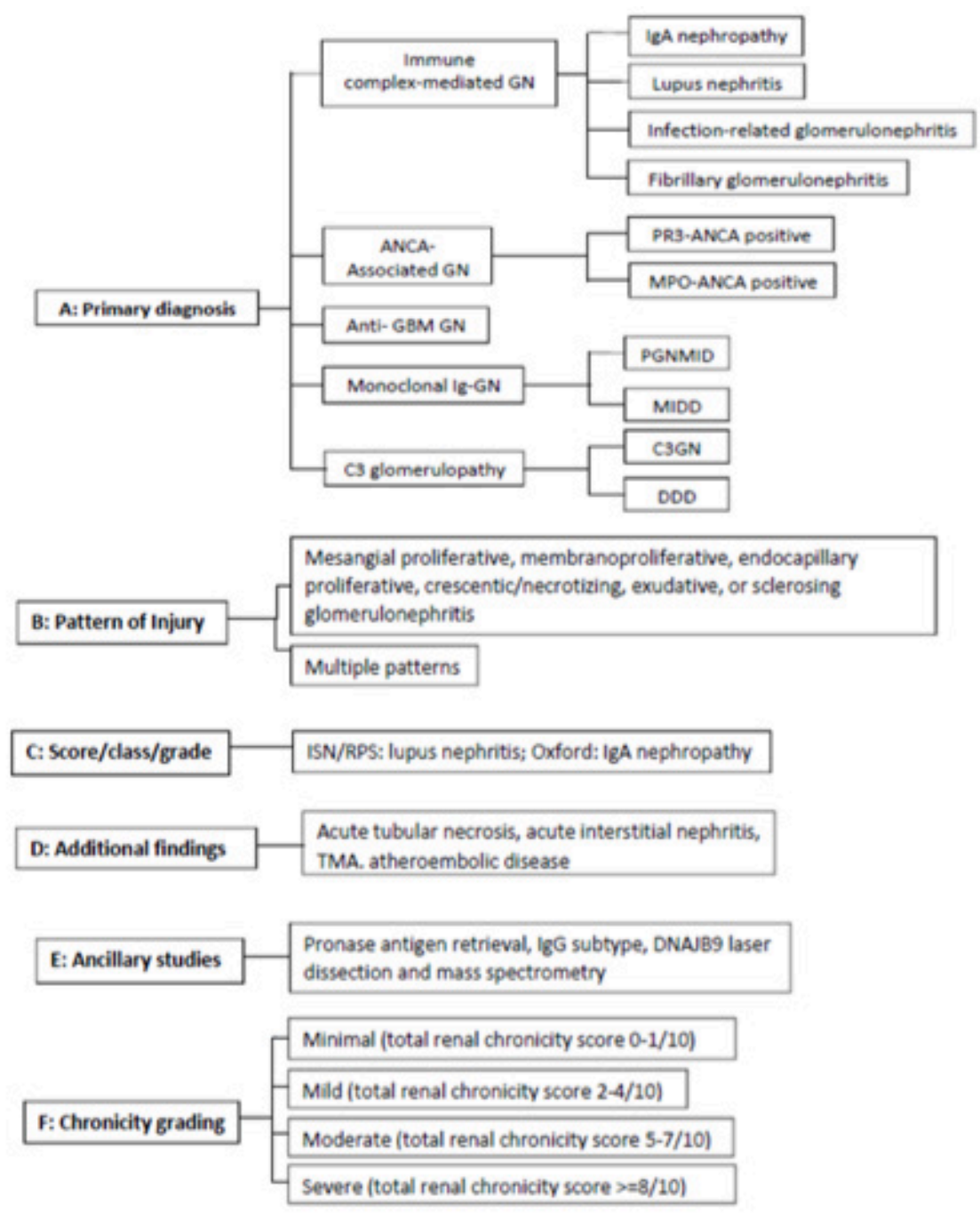

Figure 4: Overview of standardized classification and reporting of GN.

glomerular efferent arterioles. Following complete glomerular sclerosis, blood flow and glomerular filtration cease, and the dependent tubules undergo both ischemic and functional atrophy with adjacent interstitial fibrosis. Such secondary tubulointerstitial changes should not be mistaken for primary tubulointerstitial disease. Likewise, primary vascular disease can cause secondary changes in glomeruli and tubules.

Sequential evaluation of four renal components:

\section{Glomeruli}

- No. of glomeruli

- $\quad$ No. of (\%) global sclerosis (if present)

- $\quad$ No. of (\%) segmental sclerosis (if present)

- No. of (\%) crescents, cellular to fibrocellular (if present)

- $\quad$ No. of (\%) fibrinoid necrosis (if present)
- Glomerular hypercellularity, deposits, thrombosis, thickening of the glomerular basement membrane, double contours, spikes

Because each LM pattern of GN can have many different causes with very different prognoses, recognition of the specific cause of the injury in a given specimen is very important. Spectrum of light microscopic morphology in response to injury is given in Figure 3. ${ }^{12}$

\section{Tubules}

- Acute injury, necrosis

- Atrophy

- Casts (RBC, protein, pigment, neutrophils)

- Tubular reabsorption droplets

- Vacuolization, nuclear inclusions

\section{Interstitium}


- Interstitial infiltrate

- $\quad$ Fibrosis

- $\quad$ Edema

\section{Vessels}

- Number of arteries

- Intimal fibrosis, medial thickening, mucinous degeneration

- Arteriolar hyalinization, fibrinoid necrosis

- Vasculitis

- Peritubular capillaritis

\section{Immunoflourescence/ Immunohistochemistry}

The numbers of glomeruli are counted. The glomerular and extra glomerular location, intensity, and pattern of staining are observed, recorded, and reported. Glomerular staining is categorized as mesangial or capillary wall or both. As with LM lesions, the distribution is described as focal or diffuse, and segmental or global. Capillary wall staining is described as granular, linear, or band-like. The location of the capillary wall staining in the GBM (intramembranous), between the GBM and the podocytes (subepithelial), or between the GBM and endothelial cells (subendothelial) sometimes can be discerned. Staining intensity, location/ pattern of staining for each antibody, and specify intensity scale $(0-3+$ or $0-4+)$

\section{Electron Microscopy}

EM allows a detailed evaluation of the cellular and extracellular contents of each glomerular compartment and assessment of the thickness, contour, and integrity of the GBM and mesangial matrix. Abnormal deposits, such as electron-dense immune deposits, or organized fibrillary or microtubular deposits, can be detected in subepithelial, intramembranous, and mesangial locations. Ultrastructural abnormalities in GBMs include thickening, thinning, lamellation, and subendothelial electron-lucent expansion. Glomerular deposits with an organized (patterned) substructure are pathognomonic in some circumstances or at least narrow the differential diagnosis substantially. Abnormalities in cells can be readily detected, such as the effacement of podocyte foot processes seen with proteinuria or the swelling of endothelial cells seen with eclampsia/ preeclampsia and the thrombotic microangiopathies.

The schema for diagnosing immune-mediated glomerular diseases based on integration of IFM EM, serology, and other laboratory and clinical data is shown in figure $4 .{ }^{13}$
GNs are classified into the five principal groups as shown in figure $5 .{ }^{14}$ In general, placement within one class means that other classes of GN can be excluded and this can then guide one toward correct evaluation, diagnosis and management of the GN. While immune complex-mediated GN is most heterogeneous and contains many specific diseases, other groups such as ANCA-associated GN, antiglomerular basement membrane (GBM) GN, monoclonal Ig GN and C3 glomerulopathy are rather specific and in themselves point to the specific etiology of the GN.

Once the LM, IF and EM findings are collected, as well as relevant clinical data an integrated interpretation must be formulated and a diagnosis, rendered. For glomerular diseases, in particular, this diagnosis often includes both terms that describe the histologic pattern of injury seen by LM and terms that reflect findings by IF or EM. The diagnosis could be further modified by knowledge of findings in the patient not observed in the renal biopsy specimen, e.g. Henoch-Schönlein purpura nephritis with focal proliferative and sclerosing GN.

Basic format of kidney biopsy report is given below. ${ }^{15,16}$

(1) Specimen type: needle biopsy, wedge, etc.

\section{(2) Diagnosis}

\section{Primary diagnosis}

Disease process/pathogenic type (e.g., IgA nephropathy, lupus GN, ANCA GN, C3 GN)

Pattern of glomerular injury (e.g., mesangial proliferative, membranoproliferative, necrotizing/crescentic, and focal and segmental sclerosing)

Histologic scores or grade (e.g., Oxford/MEST for IgA nephropathy and ISN/RPS for lupus nephritis)

Additional features(e.g., degree ofglobalglomerulosclerosis, IFTA, vascular sclerosis, clinical modifiers, such as cryoglobulin/clinical HCV, bacterial endocarditis/clinical, staphylococcal cellulitis/clinical)

Secondary diagnoses (list; e.g., acute interstitial nephritis and diabetic glomerulosclerosis); these are not felt to be part of the primary disease

\section{(3) Comment/narrative}

Can be used for summarizing/clarifying morphologic basis of primary and/or secondary diagnoses or clinicopathologic correlations, providing prognostic information, discussing differential diagnosis, and providing appropriate references

(4) Summary of clinical data 
(5) Gross description

(6) LM description-stains or IHC performed, presence of cortex/medulla, four histological compartments

\section{(7) $\mathrm{IF} / \mathrm{IHC}$}

(8) EM

(9) Addendum for special studies

\section{CONCLUSIONS}

The diagnosis of glomerular disease in renal biopsy consists of 5 steps that may occur in different sequences: 1) preliminary review of available clinical data prior to specimen examination, 2) LM examination, 3) IF/IHC examination, 4) EM examination, and 5) integration of all pathologic and clinical data into a final diagnosis. Standardization of the renal biopsy report of GN reinforces the systematic approach to the evaluation of the biopsy and may increase diagnostic accuracy.

\section{Conflict Interest: None}

\section{REFERENCES}

1. Couser WG. Basic and translational concepts of immune-mediated glomerular diseases. J Am Soc Nephro 2012;23:381. Crossref

2. Anders HJ, Fogo AB. Immunopathology of lupus nephritis.Semin Immunopathol. 2014;36:443. $\underline{\text { Crossref }}$

3. Radhakrishnan J, Perazella MA. Drug-induced glomerular disease: attention required. Clin J Am Soc Nephrol. 2015;10:1287. Crossref

4. Jhaveri KD, Shah HH, Calderon K, Campenot ES, Radhakrishnan J. Glomerular diseases seen with cancer and chemotherapy: a narrative review. Kidney Int. 2013;84:34. Crossref

5. Van den Berg JG, Weening JJ. Role of the immune system in the pathogenesis of idiopathic nephrotic syndrome. Clin Sci (Lond). 2004;107:125. Crossref
6. Viswanathan G, Upadhyay A. Assessment of proteinuria. Adv Chronic Kidney Dis. 2011;18(4):243-8. Crossref

7. Montañés Bermúdez R, Gràcia García S, Pérez Surribas D, et al. Consensus document. Recommendations on assessing proteinuria during the diagnosis and follow-up of chronic kidney disease. Nefrologia. 2011;31(3):331-45. Crossref

8. National Kidney Foundation. KDOQI clinical practice guidelines for chronic kidney disease: evaluation, classification, and stratification. Am J Kidney Dis. 2002;39(2 suppl 1):S1-266. Crossref

9. Luciano RL, Moeckel GW. Update on the Native Kidney Biopsy: Core Curriculum 2019. Am J Kidney Dis. 2019;73:404-15. Crossref

10. Colvin R, Chang A. Introduction to renal pathology, in Diagnostic Pathology: Kidney Diseases. 2nd Edition. Elsevier: 2015.pp4

11. Agnes Fogo Michael Kashgarian.Diagnostic Atlas of Renal Pathology 3rd Edition. Elsevier. In: approach to diagnosis of the kidney biopsy 2016 pp6.

12. Jennette JC, Silva FG, Olson JL, D'Agat VD.Primer on the Pathologic Classification and Diagnosis of Kidney Disease, In Heptinstall's Pathology of the Kidney.7th edition. Lippincott Williams \& Wilkins;2014. Pp 91-117

13. Jennette JC, Falk RJ. Diagnosis and management of glomerular diseases. Med Clin North Am 1997;81:653. Crossref

14. Sethi S and Fervenza FC . Standardized classification and reporting of glomerulonephritis. Nephrol Dial Transplant (2018) 1-6. Crossref

15. Chang A, Gibson IW, Cohen AH, Weening JJ, Jennette JC, Fogo AB. A position paper on standardizing the nonneoplastic kidney biopsy report. Clin J Am Soc Nephrol 2012;7:1365-8. Crossref

16. Sethi S et al. Mayo Clinic / Renal Pathology Society Consensus Report on Pathologic Classification, Diagnosis, and Reporting of GN. J Am Soc Nephro. 2016;(5):1278-87. Crossref 